\title{
A rapid MCM-41 dispersive micro-solid phase extraction coupled with $L C / M S / M S$ for quantification of ketoconazole and voriconazole in biological fluids
}

\begin{abstract}
A rapid dispersive micro-solid phase extraction (D- $\varepsilon$-SPE) combined with LC/MS/MS method was developed and validated for the determination of ketoconazole and voriconazole in human urine and plasma samples. Synthesized mesoporous silica MCM-41 was used as sorbent in $d-\varepsilon-S P E$ of the azole compounds from biological fluids. Important D- $\varepsilon$-SPE parameters, namely type desorption solvent, extraction time, sample $\mathrm{pH}$, salt addition, desorption time, amount of sorbent and sample volume were optimized. Liquid chromatographic separations were carried out on a Zorbax SB-C18 column $(2.1$ 姆植00 Thm, 3.5 [ d $\mathrm{dm}$ ), using a mobile phase of acetonitrileï $0.05 \%$ formic acid in $5 \mathrm{Th} / \mathrm{m}$ ammonium acetate buffer $(70: 30, \mathrm{v} / \mathrm{v})$. A triple quadrupole mass spectrometer with positive ionization mode was used for the determination of target analytes. Under the optimized conditions, the calibration curves showed good linearity in the range of $0.11110,000$ tdg/L with satisfactory limit of detection $(\hat{O} .06[\mathrm{lg} / \mathrm{L})$ and limit of quantitation $(\hat{O} \mathrm{O} .3[\mathrm{fg} / \mathrm{L})$. The proposed method also showed acceptable intra- and inter-day precisions for ketoconazole and voriconazole from urine and human plasma with RSD Ò $6.5 \%$ and good relative recoveries in the range 84.3ت̈ $114.8 \%$. The MCM-41-D- $\varepsilon-S P E$ method proved to be rapid and simple and requires a small volume of organic solvent (200[द्dL); thus it is advantageous for routine drug analysis.
\end{abstract}

Keyword: Dispersive micro-solid phase extraction; Ketoconazole; LC/MS/MS; MCM-41; Voriconazole 